\title{
HUMAN ACTIVITY RECOGNITION AND FALL DETECTION
}

\author{
Disha Deepak Jatkar \\ Department of Computer Science and Engineering \\ Walchand College of Engineering \\ (An Autonomous Institute), \\ Sangli, India
}

\begin{abstract}
Personalized monitoring and its application is increasing with the advancement of technology. And during pandemics its become very essential to keep an eye on the prone area and one of the area to identify was old age home. Dizziness, unconsciousness, and others are the common problems associated with elderly people due to weakness and this was also the symptoms of covid. So an unusual activity of falling of elderly people was very difficult to identify and also to monitor. The technology was updated till now to identify posture of normal activity such as running, walking, jumping and many but revert to that falling was an area need to explore. During the fall of an elderly person, the injuries are very fatal, and to void this case we proposed a design to identify the fall and try to notify the system about its fall. Although we try to predict the fall so that it becomes easy to monitor and provide medical help as soon as possible. The main theme is to identify the posture activity and once identify we will compare the activity with trained datasets and if it's normal in vision them no notification occurred and if the percentage of falls was high then we can predict the system as fall video.
\end{abstract}

Keywords- Elderly People, Activity Detection, Fall Detection, Predicted.

\section{INTRODUCTION}

Many elderly are living alone during this lockdown and can have limited access to various facilities especially to medical help also. Various methods of technology has evolves to identify and monitor the activities related with it. And hence to monitor further about its fall it's very essential and important to predict the fall of a person.

A surveillance camera will be available to capture the activity of elderly people and feed continuous video into the system for training part and for modeling purpose. There are two procedures to follow, first one is to feed those videos to the activity model and second step is to compare the activity frames with dataset and predict the fall. People above 65 age have the problem of weaker muscle and may lead to collapsing which affect critical injuries. An unusual activity model was creates to predict the fall with reference to the

\author{
Prof. Anil R. Surve \\ Department of Computer Science and Engineering \\ Walchand College of Engineering \\ (An Autonomous Institute), \\ Sangli, India
}

video feed. It will definitely help to resolve the issue of unusual activity and estimate the fall quickly and efficiently [1]. The World Health Organization, falls are defined as an event in which a person will rest on the ground or at any lower level. There are an estimated 646,000 fatal accidents every year, the second leading cause of death from accidental injuries, after road injuries. Worldwide, mortality rates are very high for adults over the age of 60 . More than $50 \%$ of trauma related hospitals are seen in people over the age of 65 . [2].

A fall also causes a hip fracture, another common problem in the elderly. According to Health Quality Ontario, the average time taken to treat an emergency at Kingston General Hospital (KGH) is 3.1 hours. With the rising cost of health care and the elderly with chronic illnesses, there is an urgent need to move elderly patients from hospitals to other care facilities such as smart retirement homes. [3].

\section{LITERATURE SURVEY}

In [5], These applications offer methods for determining if a person is walking, running, jumping, jogging, or falling, among other actions. Fall detection is especially significant among these activities because it is a common and dangerous event for people of all ages, with a disproportionately adverse effect on the elderly. Sensors are typically used in these applications to detect abrupt changes in a person's movement.

Post measurement is introduced as a feature removal process that allows RGB images to be represented by human bone sets, with the most prominent bones in each image being considered. [5]

Shobhanjana Kalita et. al. Extended CORE9 was utilized to provide a qualitative spatial description of the video activity for human activity recognition. A graph structure may be used to encode the spatial description of an activity acquired using Extended CORE9 as well as the time information. For human-human and human-object interactions, extended CORE9 has been used. They demonstrate how Extended CORE9 may be used for singleperson tasks in this article.

For human-human or human-object interactions, including geographical information within a graph 
representation gives classification results that are similar to the state-of-the-art [2].

Two steps are followed in this system: Only the acceleration data can be utilized for activity recognition in this system, which increases classification accuracy by extracting features from the autocorrelation function and the power spectral density of both the acceleration and angular velocity data.

It is worth noting that the fall detection accuracy for QSVM and EBT is $100 \%$ with no false alarms, which is the greatest possible performance [4].

Falls can be noticed in elderly individuals who live alone in their homes, and a disabled person may experience specific occurrences such as falling, making it necessary to watch the actions of the elderly. This study introduces the accelerometer sensor and RFID technology, which may be used to track the activities of senior citizens.

To improve performance and recognise diverse actions, use the KNN classifier. Future study will concentrate on developing a device-free activity recognition system for the elderly, based on the use of inconspicuous, low-cost passive RFID tags. [3]

Two wearable accelerometers, stitched inside elastic sportswear and positioned on the belly and right thigh, make up the system. On a laptop, the raw sensor data received over Bluetooth is used to recognize the user's actions and to detect falls. The RAReFall system incorporates two accelerometers inside elastic sportswear, which are worn on the abdomen and right thigh, respectively. The AR and FD are carried out on a laptop with raw sensor data obtained through Bluetooth.

It was intended for real-world performance, therefore it employs a combination of rather mature yet carefully tuned techniques. Because the competition environment is more realistic than most $A R$ assessments, our performance at the competition demonstrates RAReFall's practical usefulness. [1]

\section{PROPOSED SYSTEM}

Create a system which will predict the fall also, minimize the fatal accident happens to elderly people due to falling. Overcome the problem associated with fall of elderly people which cause a critical injuries or other. Then track the activity of elderly people by continuous monitoring. Visionbased devices have the same drawback as ambient devices: they have to be installed in multiple rooms to cover a larger area of interest. Another challenge is the treatment of privacy because images from a real person's life are involved. Privacy preserving Deep Learning.

A solution is a system that processes all visuals on the edge (Edge Computing), does not send any images at all but sends an alert when the fall has been detected. Another option is sending images only when the fall has been detected (Edge AI with data offloading), those images can be blurred easily to avoid facial recognition from third parties (Face Blur).
Recently, several approaches for privacy-preserving deep learning have been introduced. There are several issues with computerized detection of falls the usage of video surveillance cameras (top-quality video adjustment, converting lighting conditions cause difficulties in picture processing, tasks human beings carry out every day with sure moves inclusive of falls). Therefore, it's miles almost not possible to build a video system with $100 \%$ acquisition accuracy. We intend to create a system as it should be hit upon in at least $85 \%$ of cases.

One of the first issues is the selection of surveillance cameras. Video recordings are taken with luxurious, superb cameras (e.G. Axis video cameras) that include a high diploma of video compression (e.G. Mpeg4) that could extract nonphotographic fabric. If the room is lit utilizing natural mild, night, and day changes, weather situations and artificial lighting fixtures will substantially affect the changes in light. These modifications must be taken into consideration while keeping apart transferring objects from a fixed domain. In addition to these modifications in light, modifications inside the heritage can occur because of transient actions that encompass for instance converting seats or moving an e-book. Such facilities ought to be included within the background. So it's far great to construct a website once in a while. Every other hassle with lights is the prevalence of thinking (shades are frequently lighter than ordinary) and the shadow of moving items (colors are darker than regular). They can reason troubles if they're observed to be shifting. Often, the first-rate hassle in segregation could be insufficient differences and differences among a person and a background, for instance, someone sporting a white robe walking in front of a white wall or a colored object on foot in the front of a crowded area within the background.

\section{METHODOLOGY}

To do the system more accurate the database needs to gather videos associated with the falling pattern. Once the data was gathered we need to monitor the falling frames and divide them into fall and not falling postures. With the help of falling frames, it becomes easy to identify the real view of falling of a person by comparing the real-time or dataset falling video with the frames we classified.

\section{1) Sequence of Execution:}

- Input data signals from dataset or real-time camera.

- Pre processing of input data to generate frames through segmentation.

- Feature are extracted for specific activity.

- Models for specific activity was created.

- The generated values were compared with an ideal value and fall detection or not was justified. 
International Journal of Engineering Applied Sciences and Technology, 2021

Vol. 6, Issue 3, ISSN No. 2455-2143, Pages 269-274

Published Online July 2021 in IJEAST (http://www.ijeast.com)

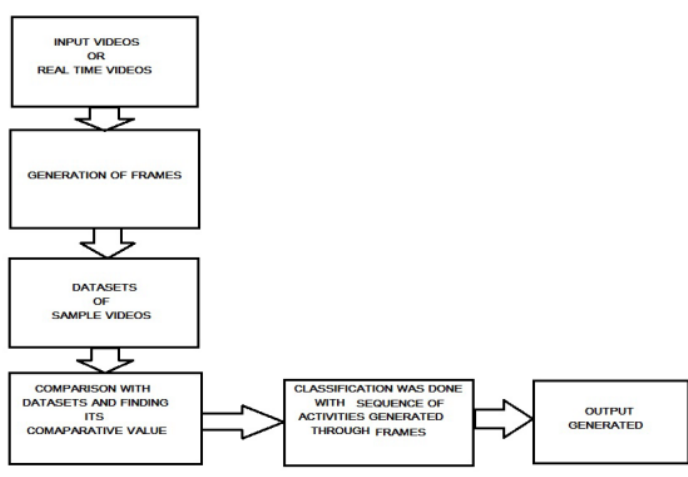

Fig. 1. Depicting the flow of the system for fall detection

\section{2) To clarify the above diagram more clearly the} following procedure was provided:

The input dataset video or a real-time video was sent to justify the fall. This video was then split down into frames, which were then scaled, turned into activity sequences, and delivered to the Activity Recognition and Fall Detection Models. The actions in the frames are recognized by the activity recognition model. From the datasets where the comparison values were obtained, this sequence of actions is then transmitted to the constructed model. This sequence of actions is classed as abnormal if these values are less than the optimum value. Fall activities need to identify so early to provide the help urgently.

\section{Activities For Recognition}

Usual activities which identified of constant posture

- Walking

- Working at Computer (working PC).

- Standing Up, Walking and Going up/down stairs.

- Standing.

- Going Up/Down Stairs (stairs)
- Clapping

- Jumping

- Running

- Seating

- $\quad$ Bending

\section{Preprocessing}

1. Conversion of videos into frames:

This process was done after classifying the videos into various frames and that frames were stored for training with input sequence. The input will be either the stored video or from a live camera. The patterns of the frames were resized for $300 * 300$ pixels.

\section{Frames Conversion:}

These resized frames were converted into a sequence of activity and extracted its features in comparison with the dataset model and help to predict the fall. The intensity of frames was different from other frames surrounding.

\section{State Classification}

The k-Nearest Neighbors (KNN) algorithm is used within the current state recognition system by topics. KNN can be a machine learning algorithm, in which a new set of information read and it is compared to the same well-known data, and the value, fall or fall, is given in all state calculations in government and distance to nearby neighbors. The KNN algorithm takes the angle, subject ratio, and three most up-todate values for ratio change speed as input. The output is either a fall or not fall state.

\section{FLOWCHART}

1. Sequence of Execution:

- Input data signals from dataset or real-time camera.

- Preprocessing of input data to generate frames through segmentation.

- Feature are extracted for specific activity.

- Models for specific activity was created. 
International Journal of Engineering Applied Sciences and Technology, 2021

Vol. 6, Issue 3, ISSN No. 2455-2143, Pages 269-274

Published Online July 2021 in IJEAST (http://www.ijeast.com)

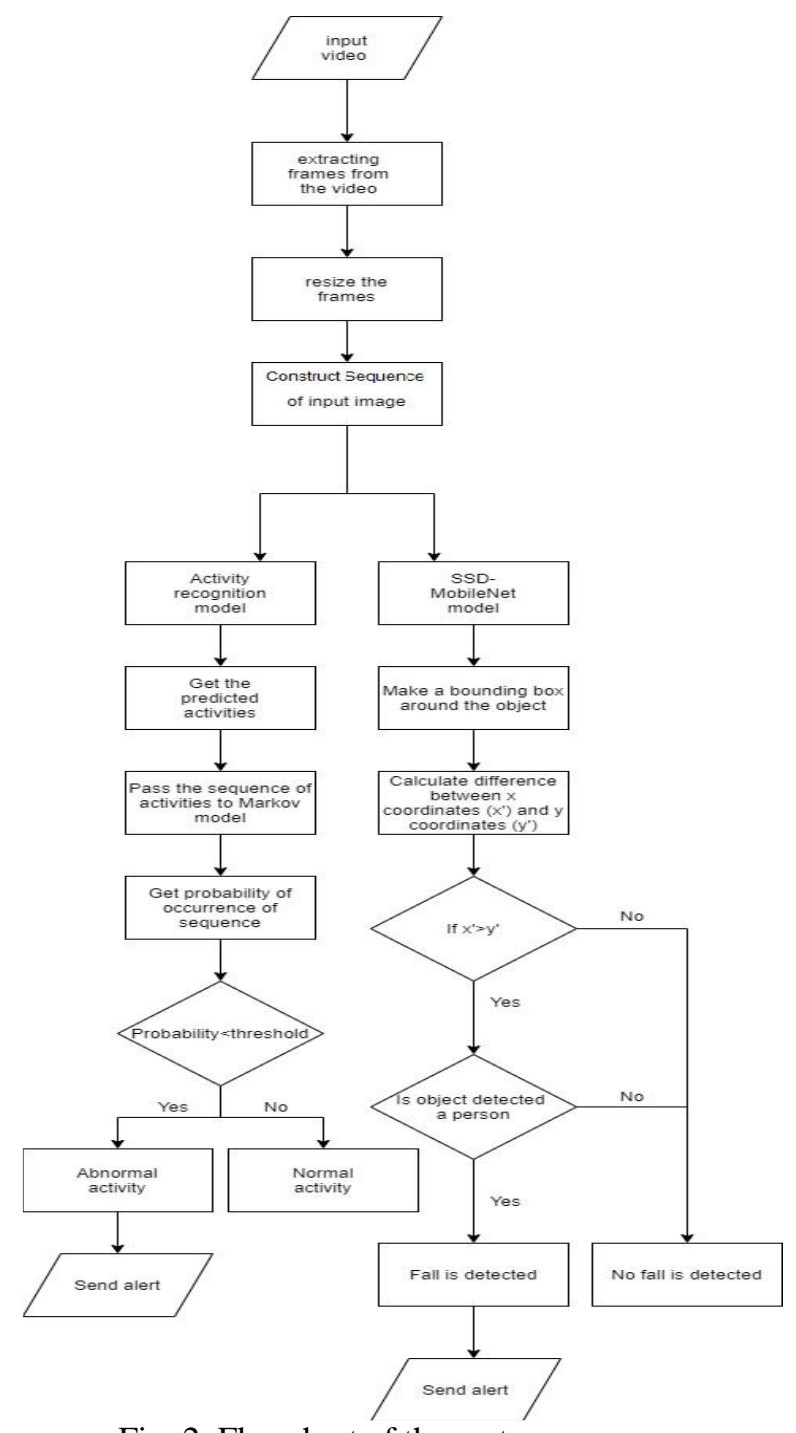

Fig. 2. Flowchart of the system

\section{RESULTS}

1) Selecting the Input :

Fig. 3 shows the selection of the input. This window helps to select the input video sequence which we need to generate the frames for training part and model creation.

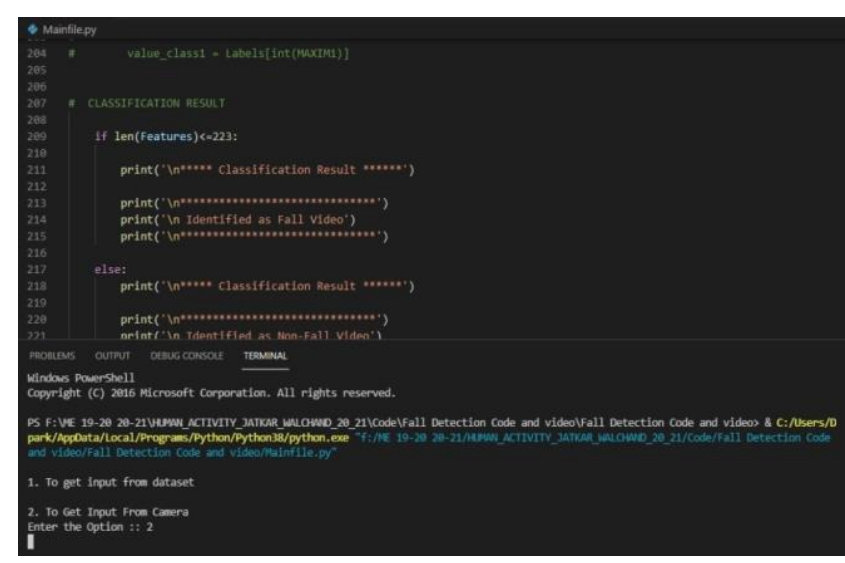

Fig.3 Selecting the input

\section{2) Selection of Video :}

Fig. 4 shows the selection of the video. The dataset consist of video stored will be selected from the specified path and the frames was generated.

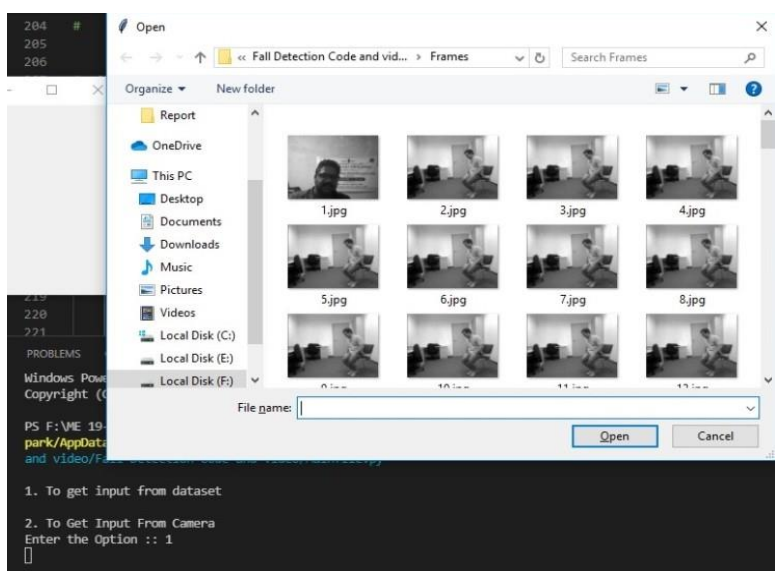

Fig.4 Selection of video

\section{3) Frames Generation :}

The selected video generates the frames that was shown in the window during execution of the video. The frame generation step shows in fig. 5 .

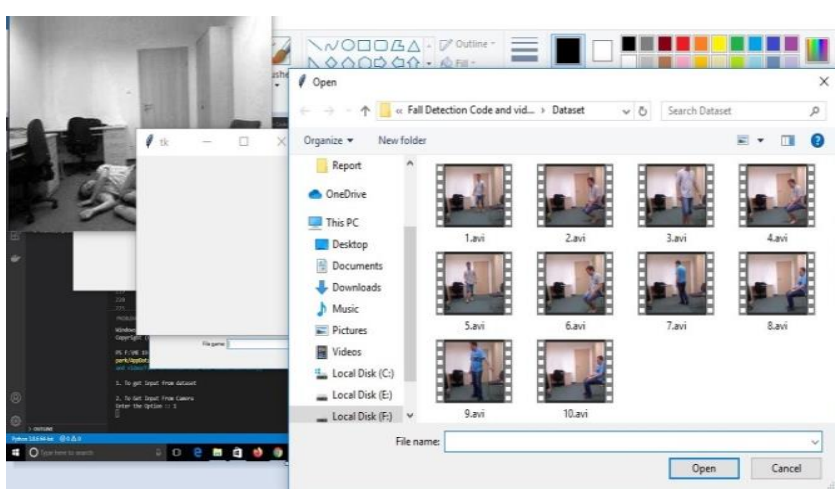

Fig. 5 Frames generation 


\section{4) Selected Frame :}

Fig. 6 shows the frame from the video to be selected to identified the fall of the person and detect as the falling video if the falling percentage was high as described.

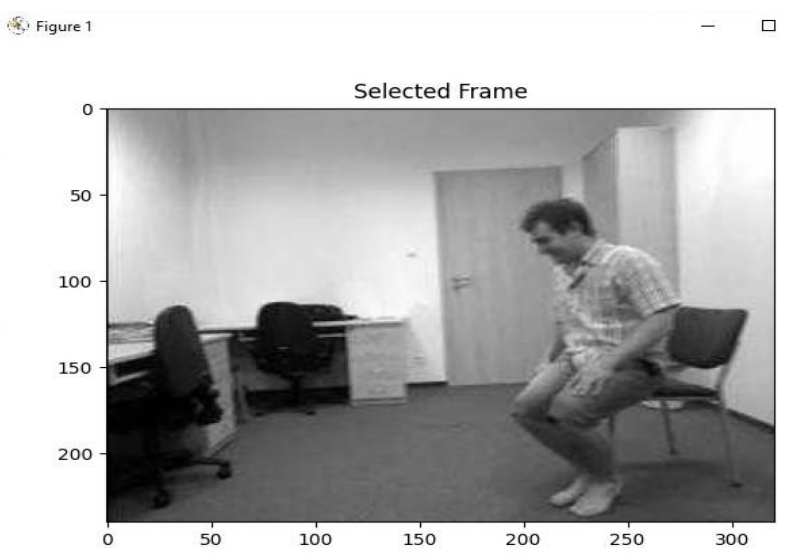

$\mathbf{\mathbf { s }}|\leftarrow| \rightarrow|+|=\mid \mathbf{b}$

Fig. 6 Selected Frames

\section{5) Feature Extraction :}

During detection of fall the extraction process was conducted so as to generate the feature classification for identification and comparison with fall features. The fig. 7 shows the result of feature extraction process.

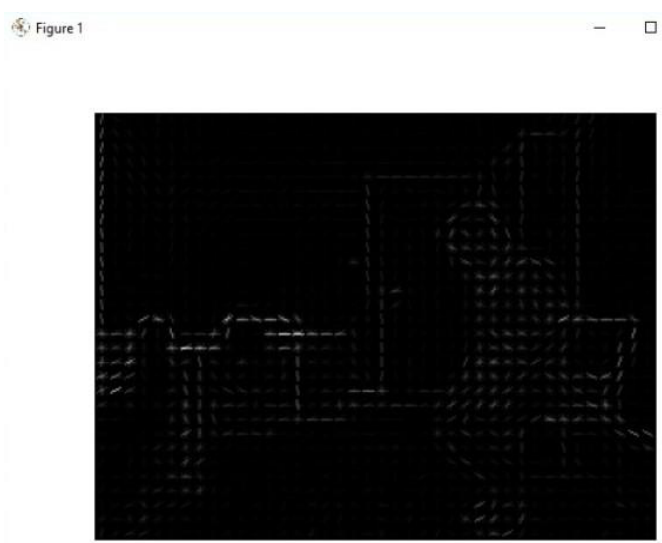

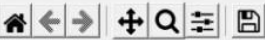

Fig. 7 Feature Extraction

\section{6) Performance Estimation :}

The accuracy, sensitivity and selectivity of the fall video was displayed as per the detection of the falling frame. Following fig. 8 gives accuracy, sensitivity and selectivity.

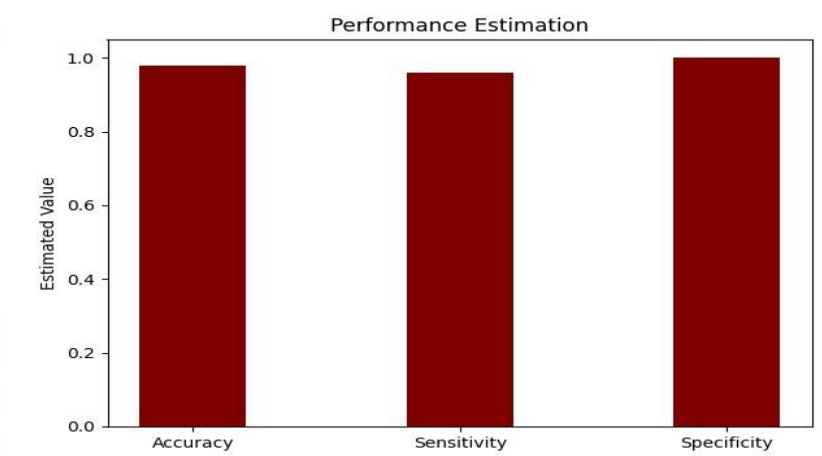

$\mathbf{N}|\leftarrow| \rightarrow| \pm \mathbf{Q}|=\mid \mathbf{b}$

Fig. 8 Performance Estimation

\section{7) Detection of Fall or Not :}

This window will display and predict the fall with highest accuracy of detection of the fall shows in fig. 9.

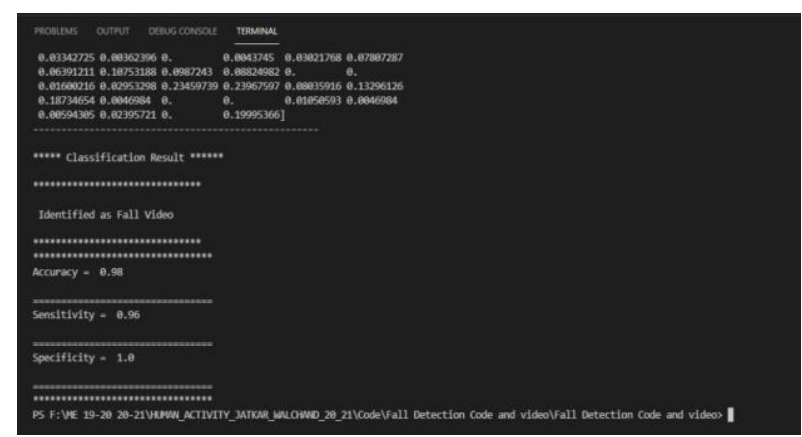

Fig. 9 Detection of fall or not

\section{CONCLUSION}

The way of living of elderly people alone during this lockdown and can have limited access to various facilities, especially to medical help also. This method is helpful to identify and monitor the activities about falling of an elderly person. The use of surveillance cameras will be available to capture the activity of elderly people and feed continuous video into the system for training part and modeling purposes. Two procedures were proposed and design of which the first one is to feed those videos to the activity model and the second one is to compare the activity frames with the dataset and predict the fall. People above 65 age have the problem of weaker muscle and may lead to collapsing which affects critical injuries. An unusual activity model was created to predict the fall with reference to the video feed. It will help to resolve the issue of unusual activity and estimate the fall quickly and efficiently. Further, it will be extended as a part of the research. 


\section{REFERENCE}

[1] Hristijan Gjoreski, Simon Kozina, Matja z Gams, Mitja Lu`strek RAReFall- Real-time Activity Recognition and Fall Detection System 2014 IEEE International Conference on Pervasive Computing and Communications Demonstrations.

[2] Shobhanjana Kalita, Arindam Karmakar, Shyamanta M Hazarika, Fall Detection during Activities of Daily Living using Extended CORE9 2019 Second International Conference on Advanced Computational and Communication Paradigms (ICACCP).

[3] Bharati Kaudki, Anil Surve , IOT Enabled Human Fall Detection Using Accelerometer and RFID Technology Proceedings of the Second International Conference on Intelligent Computing and Control Systems (ICICCS 2018).

[4] Ali Chelli, Member, IEEE, and Matthias P"atzold , IEEE Xplore Compliant Part Number: CFP18K74-ART; ISBN:9781-5386-2842-3 A Machine Learning Approach for Fall Detection and Daily Living Activity Recognition IEEE DOI 10.1109/ACCESS.2019.2906693, IEEE Access

[5] Heilym Ramirez 1, Sergio A. Velastin2,3, (Senior Member, Ieee), Ignacio Meza1, Ernesto Fabregas 4, Dimitrios Makris5, And Gonzalo Farias Fall Detection and Activity Recognition Using Human Skeleton Features, Received February 4, 2021, accepted February 13, 2021, date of publication February 23, 2021, date of current version March 3, 2021, Digital Object Identifier 10.1109/ACCESS.2021.3061626

[6] H. Gjoreski, B. Kalǔza, M. Gams, R. Mili'c, M. Lu`strek. "Ensembles of multiple sensors for human energy expenditure estimation," Proceedings of the 2013 ACM international joint conference on Pervasive and Ubiquitous computing, Ubicomp, pp. 359-362, 2013.

[7] M. J. Hall, L. Fingerhut, M. Heinen, "National Trend Data on Hospitalization of the Elderly for Injuries, 1979-2001. American Public Health Association (APHA), 2004.

[8] M. E. Tinetti,C. S. Williams, "Falls, Injuries Due to Falls, and the Risk of Admission to a Nursing Home," The New England Journal of Medicine, vol. 337, pp. 1279-1284, 1997.

[9] D. Wild, U. S. Nayak, B. Isaacs, "How dangerous are falls in old people at home?," British Medical Journal (Clinical Research Edition), vol. 282, no. 6260, pp. 266-268, 1982.

[10] EvAAL competition. http://evaal.aaloa.org/ [Accessed: November, 2013]

[11] H. Gjoreski, M. Lu`strek, M. Gams, "Accelerometer Placement for Posture Recognition and Fall Detection," In The 7th International Conference on Intelligent Environments, pp. 47-54, 2011.

[12] S. Kozina, H. Gjoreski, M. Gams, M. Lu`strek,"Threelayer Activity Recognition Combining Domain Knowledge and Meta-classification," Journal of Medical and Biological Engineering, vol 33, no 4, 2013.

[13] Shimmer sensor platform. http://www.shimmerresearch.com [Accessed: November, 2013]
[14] S. Kozina, H. Gjoreski, M. Gams, M. Lu`strek, "Efficient Activity Recognition and Fall Detection Using Accelerometers," Evaluating AAL Systems Through Competitive Benchmarking Communications in Computer and Information Science, Volume 386, pp 13-23, 2013.

[15] H. Gjoreski, M. Lu`strek, M. Gams.: Context-Based Fall Detection using Inertial and Location Sensors. In: International Joint Conference on

Ambient Intelligence, Lecture notes in computer science, pp. 1-16, 2012. 2014 IEEE International Conference on Pervasive Computing and Communications Demonstrations

[16] Riya Dichwalkar , Shreya Oak , Tania Rajabally , and Dhananjay Kalbande ," Activity Recognition And Fall Detection In Elderly People," 11th ICCCNT 2020 July 1-3, 2020 - IIT - Kharagpur, IEEE - 49239

[17] Yoosuf Nizam , Mohd Norzali Haji Mohd, and M. Mahadi Abdul Jamil ," Development of a User-Adaptable Human Fall Detection Based on Fall Risk Levels Using Depth Sensor," doi:10.3390/s18072260

[18] Djamila Romaissa Beddiar1, Brahim Nini1 ,Mohammad Sabokrou, Abdenour Hadid." Vision-based human activity recognition: a survey," Vision-based human activity recognition: a survey. 